\title{
Unusual competition of superconductivity and charge-density-wave state in a compressed topological kagome metal
}

\section{Fanghang $\mathrm{Yu}$}

University of Science and Technology of China

\section{Donghui Ma}

University of Science and Technology of China

\section{Weizhuang Zhuo}

University of Science and Technology of China

\section{Shiqiu Liu}

University of Science and Technology of China

\section{Xikai Wen}

University of Science and Technology of China

\section{Bin Lei}

University of Science and Technology of China

\section{Jianjun Ying}

University of Science and Technology of China

Xianhui Chen ( $\nabla$ chenxh@ustc.edu.cn )

University of Science and Technology of China https://orcid.org/0000-0001-6947-1407

\section{Article}

Keywords: Nontrivial Topology of Band Structures, High-pressure Electrical Transport, Double-peak Behavior, Lifshitz Transition of Fermi Surface

Posted Date: March 15th, 2021

DOl: https://doi.org/10.21203/rs.3.rs-285262/v1

License: (1) This work is licensed under a Creative Commons Attribution 4.0 International License. Read Full License

Version of Record: A version of this preprint was published at Nature Communications on June 10th, 2021. See the published version at https://doi.org/10.1038/s41467-021-23928-w. 
Unusual competition of superconductivity and charge-density-wave state in a compressed topological kagome metal

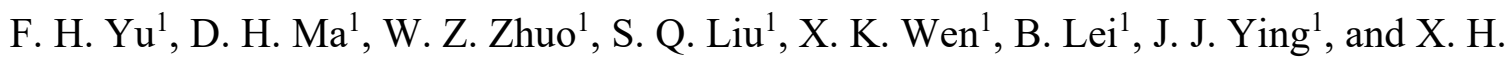 \\ Chen ${ }^{1,2,3}$ \\ ${ }^{1}$ Hefei National Laboratory for Physical Sciences at Microscale and Department of Physics, and CAS Key \\ Laboratory of Strongly-coupled Quantum Matter Physics, University of Science and Technology of China, \\ Hefei, Anhui 230026, China \\ ${ }^{2}$ CAS Center for Excellence in Quantum Information and Quantum Physics, Hefei, Anhui 230026, China \\ ${ }^{3}$ Collaborative Innovation Center of Advanced Microstructures, Nanjing 210093, People's Republic of \\ China
}

Understanding the competition between superconductivity and other ordered states (such as antiferromagnetic or charge-density-wave (CDW) state) is a central issue in condensed matter physics. The recently discovered layered kagome metal $A \mathrm{~V}_{3} \mathrm{Sb}_{5}(A=\mathrm{K}, \mathrm{Rb}$, and $\mathrm{Cs})$ provides us a new playground to study the interplay of superconductivity and CDW state by involving nontrivial topology of band structures. Here, we present high-pressure electrical transport measurements for $\mathrm{CsV}_{3} \mathrm{Sb}_{5}$ with the highest $T_{\mathrm{c}}$ of $2.7 \mathrm{~K}$ in $A \mathrm{~V}_{3} \mathrm{Sb}_{5}$ family. The $\mathrm{CDW}$ transition is monotonically suppressed by pressure, while superconductivity is enhanced with increasing pressure up to $\mathrm{P} 1 \approx 0.7 \mathrm{GPa}$, then an unexpected suppression on superconductivity happens until pressure around 1.2 GPa. The CDW is completely suppressed at a critical pressure P2 $\approx 2$ GPa together with a maximum $T_{c}$ of about $8 \mathrm{~K}$. In contrast to a common domelike behavior, the pressure-dependent $T_{c}$ shows an unexpected double-peak behavior. The unusual suppression of $T_{\mathrm{c}}$ at $\mathrm{P} 1$ is linked to a Lifshitz transition of Fermi surface evidenced by quantum oscillation experiment, a sudden enhancement of the residual resistivity and a rapid decrease of magnetoresistance. A possible nearly commensurate CDW state involving the formation of CDW domain wall has been used to account for the Lifshitz transition. Our discoveries indicate an unusual competition between superconductivity and CDW state in pressurized kagome lattice. 
Superconductivity and the charge density wave (CDW) state are two different cooperative electronic states, and both of them are originated from electron-phonon coupling and Fermi surface instabilities. Superconductivity is often observed in CDW materials, however, the interplay between CDW order and superconductivity is still not well elucidated ${ }^{1-5}$. For example, the maximum $T_{c}$ in compressed $\mathrm{TiSe}_{2}$ or $\mathrm{Cu}_{\mathrm{x}} \mathrm{TiSe}_{2}$ is reached when the CDW order is completely suppressed ${ }^{2,6}$; however, the superconductivity in compressed $1 T-\mathrm{TaS}_{2}$ and $2 \mathrm{H}-\mathrm{NbSe}_{2}$ seems to be independent of the CDW order $^{7,8}$.

The materials with kagome lattice provide a fertile ground to study the frustrated, novel correlated and topological electronic states owing to unusual lattice geometry ${ }^{9-12}$. Recently, a new family of quasi two-dimensional kagome metals $A \mathrm{~V}_{3} \mathrm{Sb}_{5}(A=\mathrm{K}, \mathrm{Rb} \mathrm{Cs})$ has attracted tremendous attentions ${ }^{13}$. These materials crystallize in the $P 6 / \mathrm{mmm}$ space group, forming layers of ideal kagome nets of $\mathrm{V}$ ions coordinated by $\mathrm{Sb}$. Besides topological properties, $A \mathrm{~V}_{3} \mathrm{Sb}_{5}$ exhibits both $\mathrm{CDW}^{13,14}$ and superconductivity ${ }^{15-17}$. Ultra-low temperature thermal conductivity measurements on $\mathrm{CsV}_{3} \mathrm{Sb}_{5}$ single crystal shows a finite residual linear term, implying an unconventional nodal superconductivity ${ }^{18}$. Intertwining the superconductivity and CDW state by involving nontrivial topology of band structures results in many exotic properties in this type of materials. For example, topological charge order was reported in $\mathrm{KV}_{3} \mathrm{Sb}_{5}{ }^{14}$, signatures of spin-triplet superconductivity were claimed in $\mathrm{Nb}-\mathrm{K}_{1-\mathrm{x}} \mathrm{V}_{3} \mathrm{Sb}_{5}$ devices ${ }^{19}$, and unconventional giant anomalous Hall effect was observed in $\mathrm{K}_{1-\mathrm{x}} \mathrm{V}_{3} \mathrm{Sb}_{5}$ and superconducting $\mathrm{CsV}_{3} \mathrm{Sb}_{5}{ }^{20,21}$. However, the superconducting transition temperature $\left(T_{\mathrm{c}}\right)$ in this system is relatively low, and its correlation with the CDW and non-trivial topological states are still not known.

High pressure is a clean method to tune the electronic properties without introducing any impurities, and pressure is often used as a control parameter to tune superconductivity and CDW state. Here, we performed high-pressure electrical transport measurements on $\mathrm{CsV}_{3} \mathrm{Sb}_{5}$ single crystals with the highest $T_{\mathrm{c}}$ of $2.7 \mathrm{~K}$ in $A \mathrm{~V}_{3} \mathrm{Sb}_{5}$ family. Maximum $T_{\mathrm{c}}$ of $8 \mathrm{~K}$ is observed at $\mathrm{P} 2 \approx 2 \mathrm{GPa}$ when CDW is completely suppressed. Strikingly, an unusual suppression on superconductivity is observed between $\mathrm{P} 1 \approx 0.7 \mathrm{GPa}$ and $\mathrm{P} 2 \approx 2 \mathrm{GPa}$. It indicates exotic enhanced competition between CDW and superconductivity in this region. These results suggest an unconventional mechanism of CDW and superconductivity in $\mathrm{CsV}_{3} \mathrm{Sb}_{5}$, which makes it a rare platform to investigate the interplay of multiple electronic orders.

We performed resistivity measurements in $\mathrm{CsV}_{3} \mathrm{Sb}_{5}$ under pressure to track the evolution of the CDW state and superconductivity in the compressed material. Temperature dependence of resistivity for $\mathrm{CsV}_{3} \mathrm{Sb}_{5}$ under various pressures is shown in Fig. 1. As shown in Fig. 1a, an anomaly 
due to the CDW transition in the resistivity is clearly visible for sample 1 with PCC. The CDW transition temperature $T^{*}$ gradually decreases with increasing the pressure, and the anomaly becomes much weaker at high pressures. We can determine the CDW transition temperature $T^{*}$ precisely in the derivative resistivity curves as shown in Fig. 1b. The anomaly clearly shifts to lower temperatures with increasing the pressure, and disappears at the pressure of $\mathrm{P} 2 \approx 2 \mathrm{GPa}$. To track the evolution of $T_{\mathrm{c}}$ with pressure, the low-temperature resistivity of $\mathrm{CsV}_{3} \mathrm{Sb}_{5}$ under various pressures is shown in Fig. 1c and 1d for sample1 with PCC and sample 2 with DAC, respectively. The $T_{\mathrm{c}}$ first increases with increasing pressure as shown in Fig. 1c, however, the superconducting transition becomes much broad with pressure larger than P1 of $\sim 0.7 \mathrm{GPa}$, like a filamentary superconductivity. It is striking that the superconducting transition becomes sharp again around P2. Meanwhile the highest $T_{\mathrm{c}}$ of $8 \mathrm{~K}$ is obtained. It should be addressed that the maximum $T_{\mathrm{c}}$ achieved at high pressure is 3 times higher than that $(2.7 \mathrm{~K})$ at ambient pressure. With further increasing the pressure, the $T_{\mathrm{c}}$ is monotonically suppressed and completely disappears above $12 \mathrm{GPa}$ as shown in Fig. 1d for sample 2 with DAC.

Figure $2 \mathrm{a}$ and $2 \mathrm{~b}$ show superconducting transition under different magnetic fields for sample 1 with PCC around P1 and P2 with magnetic field applied along c axis, respectively. Much higher magnetic field is needed to suppress superconductivity around P1 and P2 compared with that at ambient pressure ${ }^{15,18}$. To investigate the evolution of $H_{\mathrm{c} 2}$ under pressure, we plot $H_{\mathrm{c} 2}$ (determined by $T_{c}^{z e r o}$ ) as a function of $T_{\mathrm{c}}$ under various pressures as shown in Fig. $2 \mathrm{c}$ and $2 \mathrm{~d}$. The $H_{\mathrm{c} 2}$ shows strong pressure dependence. $H_{\mathrm{c} 2}$ dramatically increases with increasing the pressure, and reaches a local maximum value around $\mathrm{P} 1$. With further increasing the pressure, the $H_{\mathrm{c} 2}$ can be rapidly suppressed as shown in Fig. 2c. When the pressure reaches $\mathrm{P} 2, H_{\mathrm{c} 2}$ dramatically increases to the maximum value, then $H_{\mathrm{c} 2}$ can be gradually suppressed with further increasing the pressure as shown in Fig. 2d.

Combing the high-pressure electrical measurements on sample 1 with PCC and sample 2 with DAC, we can map out the phase diagram of $\mathrm{CsV}_{3} \mathrm{Sb}_{5}$ with pressure as shown in Fig. $3 \mathrm{a}$ and $3 \mathrm{~b}$. The CDW is monotonically suppressed with increasing pressure, and the maximum $T_{c}$ locates at the end point of CDW. A subsequent monotonic reduction of $T_{c}$ is followed at higher pressure. The relatively high $T_{\mathrm{c}}(8 \mathrm{~K})$ achieved at high pressure makes it the record in the kagome lattice materials. Such competition between CDW state and superconductivity is usual since the gap opening at CDW state would dramatically reduce the density of states at Fermi surfaces, leading to the suppression of superconductivity within Bardeen-Cooper-Schrieffer (BCS) scenario. It is striking that the superconducting transition becomes much broad with pressure between $\mathrm{P} 1$ and $\mathrm{P} 2$, and $T_{\mathrm{c}}$ together 
with $H_{\mathrm{c} 2}$ are strongly suppressed. The $T^{*}$ shows a weak anomaly around $\mathrm{P} 1$, which suggests the emergence of a new CDW state. In this new CDW state, the superconductivity shows much stronger competition with $\mathrm{CDW}$ order. It will be discussed later. We can estimate the $H_{c 2}$ at zero temperature $\left(H_{\mathrm{c} 2}(0)\right)$ by linear extrapolate the $H_{c 2}$ vs $T_{c}$ curves as shown in Fig. $2 \mathrm{c}$ and $2 \mathrm{~d}$. The pressure dependence of the extracted $H_{\mathrm{c} 2}(0)$ is shown in Fig.3c, and two peaks are clearly located at P1 and $\mathrm{P} 2$. The magnitude of $H_{\mathrm{c} 2}(0)$ at $\mathrm{P} 1$ and $\mathrm{P} 2$ is one order larger than that at ambient pressure.

The pressure dependence of $T_{c}^{\text {zero }}$ clearly shows that two peaks locate at $\mathrm{P} 1$ and $\mathrm{P} 2$, and the superconducting transition width of $\Delta T_{\mathrm{c}}$ shows sudden enhancement with pressure between P1 and P2 as shown in Fig. 4a. In order to unveil the unusual suppression of superconductivity between P1 and $\mathrm{P} 2$, we plot the residual resistivity and residual-resistivity ratio (RRR) as a function of pressure as shown in Fig. 4b. The residual resistivity suddenly increases around P1 and keeps at a relatively high value with the pressure between P1 and P2. Above P2, the residual resistivity sudden drops. RRR also exhibits reduction with pressure between P1 and P2, indicating the enhanced electron scattering in this region. The magnetoresistance (MR) measured under magnetic field of 9 Tesla and at the temperature of $10 \mathrm{~K}$ shows sudden drops around $\mathrm{P} 1$. In addition, the magnetoresistance of the low-field region at $10 \mathrm{~K}$ evolves from "V" shape to "U" shape at P2 as shown in Fig. S2 in the supplementary information. However, the room-temperature resistivity gradually decreases with increasing the pressure, and does not show any anomaly at P1 and P2 as shown in Fig. 4c. These results indicate that the transitions at P1 and P2 are related to the CDW transitions rather than the normal state change at high temperature. The Shubnikov-de Haas $(\mathrm{SdH})$ quantum oscillations (QOs) measurements at $2 \mathrm{~K}$ indicate a sudden change of frequencies around P1 as shown in Fig. S3 in the supplementary information, which gives an evidence for a Lifshitz transition at $\mathrm{P} 1$ in the $\mathrm{CDW}$ state. These discoveries clearly indicate that a new CDW state emerges at low temperature at $\mathrm{P} 1$ and such new $\mathrm{CDW}$ state disappears at $\mathrm{P} 2$.

Although superconductivity was reported in some kagome lattice materials ${ }^{22,23}$, the previously reported $T_{\mathrm{c}}$ is quite low. Our high-pressure work demonstrates that the $T_{\mathrm{c}}$ in this V-based kagome material can be relatively high and easily tuned. Further enhancement of $T_{c}$ should be possible in this type of materials by using the other methods to destroy the CDW state, such as chemical substitution or electrical gating. The maximum $T_{\mathrm{c}}$ locates at the end point of CDW, which resembles many other CDW materials ${ }^{2,6}$, indicating the competition between superconductivity and CDW state. The most interesting discovery in this work is the region between P1 and P2, in which the CDW and superconductivity have much stronger competition, leading to the dramatically suppression of $T_{\mathrm{c}}$ and increment of superconducting transition width $\left(\Delta T_{\mathrm{c}}\right)$. In addition, our QO 
measurement indicates a Lifshitz transition at P1, possibly due to the formation of a new CDW state. One possibility is that the original modulation pattern may change under pressure, and a new commensurate CDW (CCDW) state or an incommensurate CDW(ICCDW) state emerges above P1. Pressure induced CCDW to ICCDW transition has been observed in the $2 \mathrm{H}-\mathrm{TaSe}_{2}{ }^{24}$. Such phase transition will dramatically modify the Fermi surface and alters $T_{\mathrm{c}}$. However, it cannot explain the superconducting transition broadening after phase transition. Another possibility is that a nearly commensurate CDW (NCCDW) state forms above P1, in which CDW domains are separated by domain walls (DW). Similar transition was also observed in $1 T-\mathrm{TaS}_{2}$ with increasing the temperature ${ }^{25}$. It is possible that the same CDW modulation pattern (David star) shows up in the CCDW state for $A \mathrm{~V}_{3} \mathrm{Sb}_{5}$. In the NCCDW state, the charge may transfer to the narrow DWs since CDW domains are partially gapped ${ }^{26}$. The enhanced interaction and scattering at the DW network will lead to the higher resistivity ${ }^{26}$. The superconductivity in the CDW domains may be strongly suppressed due to the reduction of carrier density, however, the superconductivity in the DWs may have higher $T_{\mathrm{c}}$, leading to the filamentary superconductivity. To be reminded, the rather broad superconducting transition in the NCCDW state actually resembles the manifestation of socalled pair-density-wave (PDW) order in high- $\mathrm{T}_{\mathrm{c}}$ cuprate superconductors ${ }^{27}$. In $\mathrm{La}_{2-\mathrm{x}} \mathrm{Ba}_{\mathrm{x}} \mathrm{CuO}_{4}$, superconductivity is greatly suppressed around 1/8 doping level due to the formation of a longranged stripe order and the final superconducting state at low temperatures is attributed to a PDW $\operatorname{order}^{28-30}$. This is very similar to our cases around the region with unusual suppression of $T_{\mathrm{c}}$. Therefore, it is speculated that a similar PDW order might also emerge in the superconducting state between P1 and P2, which needs further evidences from spatial-resolved spectroscopy.

In conclusion, we systematically investigate the high-pressure transport properties of the newly discovered topological kagome metal $\mathrm{CsV}_{3} \mathrm{Sb}_{5}$. When $\mathrm{CDW}$ is completely suppressed, the maximum $T_{\mathrm{c}}$ up to $8 \mathrm{~K}$ can be reached, and is three times higher than that $(2.7 \mathrm{~K})$ at ambient pressure. More interestingly, superconductivity shows an unusual suppression with pressure between P1 and P2. A Lifshitz transition occurs accompanied with the sudden enhancement of residual resistivity and the rapid decrease of magnetoresistance above P1 in the CDW state. We propose a possible picture of CCDW to NCCDW transition occurred at P1. The formation of CDW DWs in NCCDW leads to the suppression of $T_{\mathrm{c}}$ and filamentary-like superconductivity.

\section{Methods}


Material syntheses. Single crystals of $\mathrm{CsV}_{3} \mathrm{Sb}_{5}$ were synthesized via a self-flux growth method similar to the previous reports ${ }^{15}$. In order to prevent the reaction of Cs with air and water, all the preparation processes were performed in an argon glovebox. After reaction in the furnace, the asgrown $\mathrm{CsV}_{3} \mathrm{Sb}_{5}$ single crystals are stable in the air. The excess flux is removed using water and millimeter-sized single crystal can be obtained.

High-pressure measurements. Piston cylinder cell (PCC) was used to generate hydrostatic pressure up to $2.4 \mathrm{GPa}$. Daphne 7373 was used as the pressure transmitting medium in PCC. The pressure values in PCC were determined from the superconducting transition of $\mathrm{Sn}^{31}$. Diamond anvil cell (DAC) was used to generate the pressure up to $12 \mathrm{GPa}$. Diamond anvils with $500 \mu \mathrm{m}$ culet and $c$-BN gasket with sample chambers of diameter $200 \mu \mathrm{m}$ were used. Four Pt wires were adhered to the sample and $\mathrm{NaCl}$ was used as the pressure transmitting medium. Pressure was calibrated by using the ruby fluorescence shift at room temperature ${ }^{32}$. Electrical transport measurements were carried out in a Quantum Design physical property measurement system.

Note added: During the preparation of this manuscript, we noticed a similar high-pressure work ${ }^{33}$.

\section{References}

1 Gabovich, A. M., Voitenko, A. I., Annett, J. F. \& Ausloos, M. Charge- and spin-densitywave superconductors. Superconductor Science and Technology 14, R1-R27 (2001).

2 Kusmartseva, A. F., Sipos, B., Berger, H., Forró, L. \& Tutiš, E. Pressure Induced Superconductivity in Pristine 1T-TiSe 2 . Physical Review Letters 103, 236401 (2009).

3 Joe, Y. I. et al. Emergence of charge density wave domain walls above the superconducting dome in 1T-TiSe 2 . Nature Physics 10, 421-425 (2014).

4 Johannes, M. D. \& Mazin, I. I. Fermi surface nesting and the origin of charge density waves in metals. Physical Review B 77, 165135 (2008).

5 Zhu, X., Cao, Y., Zhang, J., Plummer, E. W. \& Guo, J. Classification of charge density waves based on their nature. Proceedings of the National Academy of Sciences 112, 2367 $2371(2015)$.

6 Morosan, E. et al. Superconductivity in $\mathrm{Cu}_{\mathrm{x}} \mathrm{TiSe}_{2}$. Nature Physics 2, 544-550 (2006).

7 Sipos, B. et al. From Mott state to superconductivity in 1T-TaS 2. Nature Materials 7, 960965 (2008).

8 Feng, Y. et al. Order parameter fluctuations at a buried quantum critical point. Proceedings of the National Academy of Sciences 109, 7224-7229 (2012). 

89, 025003 (2017).

10 Han, T.-H. et al. Fractionalized excitations in the spin-liquid state of a kagome-lattice antiferromagnet. Nature 492, 406-410 (2012).

11 Sachdev, S. Kagome- and triangular-lattice Heisenberg antiferromagnets: Ordering from quantum fluctuations and quantum-disordered ground states with unconfined bosonic spinons. Physical Review B 45, 12377-12396 (1992).

12 Mazin, I. I. et al. Theoretical prediction of a strongly correlated Dirac metal. Nature Communications 5, 4261 (2014).

13 Ortiz, B. R. et al. New kagome prototype materials: discovery of $\mathrm{KV}_{3} \mathrm{Sb}_{5}, \mathrm{RbV}_{3} \mathrm{Sb}_{5}$, and $\mathrm{CsV}_{3} \mathrm{Sb}_{5}$. Physical Review Materials 3, 094407 (2019).

14 Jiang, Y.-X. et al. Discovery of topological charge order in kagome superconductor $\mathrm{KV}_{3} \mathrm{Sb}_{5}$. Preprint at http://arxiv.org/abs/2012.15709 (2020).

15 Ortiz, B. R. et al. $\mathrm{CsV}_{3} \mathrm{Sb}_{5}$ : A $\mathrm{Z}_{2}$ Topological Kagome Metal with a Superconducting Ground State. Physical Review Letters 125, 247002, doi:10.1103/PhysRevLett.125.247002 (2020).

16 Ortiz, B. R. et al. Superconductivity in the $\mathrm{Z}_{2}$ kagome metal $\mathrm{KV}_{3} \mathrm{Sb}_{5}$. Preprint at http://arxiv.org/abs/2012.09097 (2020).

17 Yin, Q. et al. Superconductivity and normal-state properties of kagome metal $\mathrm{RbV}_{3} \mathrm{Sb}_{5}$ single crystals. Preprint at http://arxiv.org/abs/2101.10193 (2021).

18 Zhao, C. C. et al. Nodal superconductivity and superconducting dome in the topological Kagome metal $\mathrm{CsV}_{3} \mathrm{Sb}_{5}$. Preprint at http://arxiv.org/abs/2102.08356 (2021).

19 Wang, Y. et al. Proximity-induced spin-triplet superconductivity and edge supercurrent in the topological Kagome metal, $\mathrm{K}_{1-\mathrm{x}} \mathrm{V}_{3} \mathrm{Sb}_{5}$. Preprint at http://arxiv.org/abs/2012.05898 (2020).

20 Yang, S.-Y. et al. Giant, unconventional anomalous Hall effect in the metallic frustrated magnet candidate, $\mathrm{KV}_{3} \mathrm{Sb}_{5}$. Science Advances 6, eabb6003 (2020).

$21 \mathrm{Yu}, \mathrm{F}$. H. et al. Concurrence of anomalous Hall effect and charge density wave in a superconducting topological kagome metal. Preprint at http://arxiv.org/abs/2102.10987 (2021).

22 Li, S., Xing, J., Tao, J., Yang, H. \& Wen, H.-H. Superconductivity in $\mathrm{Ba}_{2 / 3} \mathrm{Pt}_{3} \mathrm{~B}_{2}$ with the Kagome lattice. Annals of Physics 358, 248-254 (2015).

$23 \mathrm{Yu}, \mathrm{F}$. H. et al. Pressure-induced superconductivity in a shandite compound $\mathrm{Pd}_{3} \mathrm{~Pb}_{2} \mathrm{Se}_{2}$ with the Kagome lattice. New Journal of Physics 22, 123013 (2020). 

Transition of the Charge-Density Wave in $2 \mathrm{H}-\mathrm{TaSe}_{2}$ at High Pressure. Physical Review Letters 45, 269-272 (1980).

25 Wilson, J. A., Di Salvo, F. J. \& Mahajan, S. Charge-density waves and superlattices in the metallic layered transition metal dichalcogenides. Advances in Physics 24, 117-201 (1975).

26 Lee, K. et al. Metal-to-insulator transition in Pt-doped $\mathrm{TiSe}_{2}$ driven by emergent network of narrow transport channels. npj Quantum Materials 6, 8 (2021).

27 Keimer, B., Kivelson, S. A., Norman, M. R., Uchida, S. \& Zaanen, J. From quantum matter to high-temperature superconductivity in copper oxides. Nature 518, 179-186 (2015).

28 Agterberg, D. F. et al. The Physics of Pair-Density Waves: Cuprate Superconductors and Beyond. Annual Review of Condensed Matter Physics 11, 231-270 (2020).

29 Li, Q., Hücker, M., Gu, G. D., Tsvelik, A. M. \& Tranquada, J. M. Two-Dimensional Superconducting Fluctuations in Stripe-Ordered $\mathrm{La}_{1.875} \mathrm{Ba}_{0.125} \mathrm{CuO}_{4}$. Physical Review Letters 99, 067001 (2007).

30 Hücker, M. et al. Stripe order in superconducting $\left.\mathrm{La}_{2-\mathrm{x}} \mathrm{Ba}_{\mathrm{x}} \mathrm{CuO} 4\right\}(0.095<\mathrm{x}<0.155)$. Physical Review B 83, 104506 (2011).

31 Jennings, L. D. \& Swenson, C. A. Effects of Pressure on the Superconducting Transition Temperatures of Sn, In, Ta, Tl, and Hg. Physical Review 112, 31-43 (1958).

32 Mao, H. K., Xu, J. \& Bell, P. M. Calibration of the ruby pressure gauge to $800 \mathrm{kbar}$ under quasi-hydrostatic conditions. Journal of Geophysical Research: Solid Earth 91, 4673-4676 (1986).

33 Chen, K. Y. et al. Double superconducting dome and triple enhancement of Tc in the kagome superconductor $\mathrm{CsV}_{3} \mathrm{Sb}_{5}$ under high pressure. Preprint at http://arxiv.org/abs/2102.09328 (2021).

\section{Acknowledgements}

This work was supported by the Anhui Initiative in Quantum Information Technologies (Grant No. AHY160000), the National Key Research and Development Program of the Ministry of Science and Technology of China (Grants No. 2019YFA0704901 and No. 2017YFA0303001), the Science 
Challenge Project of China (Grant No. TZ2016004), the Key Research Program of Frontier Sciences, CAS, China (Grant No. QYZDYSSWSLH021), the Strategic Priority Research Program of the Chinese Academy of Sciences (Grant No. XDB25000000), the National Natural Science Foundation of China (Grants No. 11888101 and No. 11534010), and the Fundamental Research Funds for the Central Universities (WK3510000011 and WK2030020031).

\section{Author contributions}

X.H.C and J.J.Y conceived and designed the experiments. F.H.Y performed high-pressure electrical transport measurements with the assistance from J.J.Y., D.H.M., W.Z.Z., S.Q.L., X.K.W and B.L.. F.H.Y synthesized the $\mathrm{CsV}_{3} \mathrm{Sb}_{5}$ single crystal. J.J.Y., F.H.Y. and X.H.C. analyzed and interpreted the data. J.J.Y. and X.H.C. wrote the manuscript. All authors discussed the results and commented on the manuscript.

\section{Additional information}

The authors declare no competing financial interests. Correspondence and requests for materials should be addressed to J.J.Y. (yingjj@ustc.edu.cn) or to X.H.C. (chenxh@ustc.edu.cn).

\section{Competing financial interests}

The authors declare no competing financial interests. 


\section{Figure captions:}

Figure 1| Temperature dependence of resistivity in $\mathrm{CsV}_{3} \mathrm{Sb}_{5}$ single crystals under high pressure. (a): Temperature dependences of resistivity under various pressures for sample 1 measured with PCC. (b): The derivative $d \rho_{x x} / d T$ curves under various pressures. The blue arrows indicate the CDW transition temperature $T^{*}$. (c) and (d): The evolution of superconducting transition temperatures under pressure for sample 1 with PCC and sample 2 with DAC. The red and green arrows represent the $T_{c}^{\text {onset }}$ and $T_{c}^{\text {zero }}$, respectively. All the curves were shifted vertically for clarity.

Figure 2| Upper critical field for $\mathrm{CsV}_{3} \mathrm{Sb}_{5}$ single crystal under various pressures. Upper critical field $\left(H_{c 2}\right)$ measurements for sample1 around P1 (a) and P2 (b) with magnetic field applied along $c$ axis. (c) and (d): The upper critical field $\mu_{0} H_{c 2}$ vs. $T_{c}$ under various pressures.

Figure 3|Phase diagram with pressure for $\mathrm{CsV}_{3} \mathrm{Sb}_{5}$ single crystal. (a): Phase diagram of $\mathrm{CsV}_{3} \mathrm{Sb}_{5}$ with pressure. CDW transition temperature $T^{*}$ gradually suppressed with increasing the pressure. $T^{*}$ shows an anomaly around P1. The inset indicates the probable deformation patterns in CCDW and NCCDW states as discussed in the text. (b): Pressure dependence of superconducting transition temperatures $T_{c}^{\text {onset }}, T_{c}^{\text {zero }}$ measured on various samples. $T_{\mathrm{c}}$ is strongly suppressed with the pressure between P1 and P2. (c): Pressure dependence of upper critical field at zero temperature, two peaks can be observed at $\mathrm{P} 1$ and $\mathrm{P} 2$.

Figure 4| Pressure dependence of residual resistivity, residual-resistivity ratio and magnetoresistance for $\mathrm{CsV}_{3} \mathrm{Sb}_{5}$ single crystal. (a): Pressure dependence of $T_{c}^{\text {zero }}$ and $\Delta T_{\mathrm{c}}$ for sample 1 with PCC. $T_{c}^{\text {zero }}$ exhibits two peaks at P1 and P2. (b): Pressure dependence of residual resistivity and residual resistivity ratio (RRR) for sample 1 with PCC. (c): Pressure dependence of magnetoresistance $(9 \mathrm{~T}, 10 \mathrm{~K})$ and room-temperature resistivity. 
Figure 1.
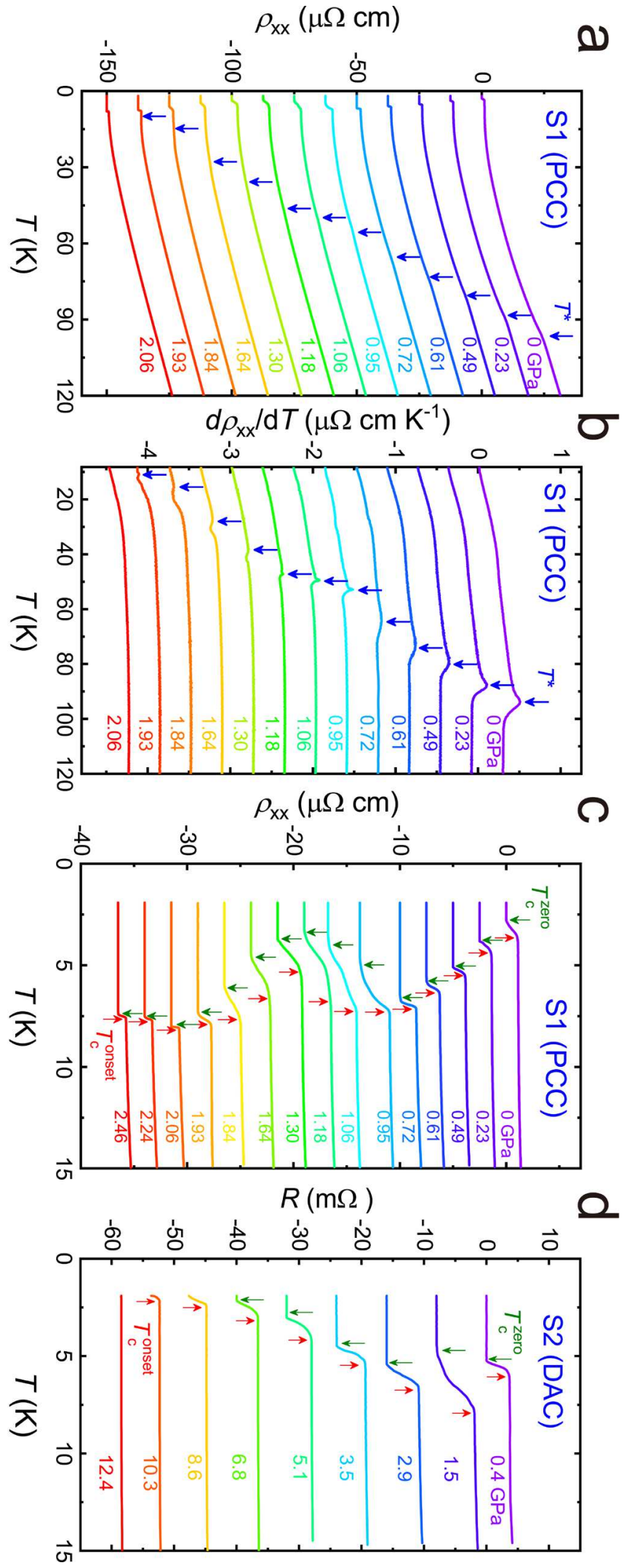
Figure 2.

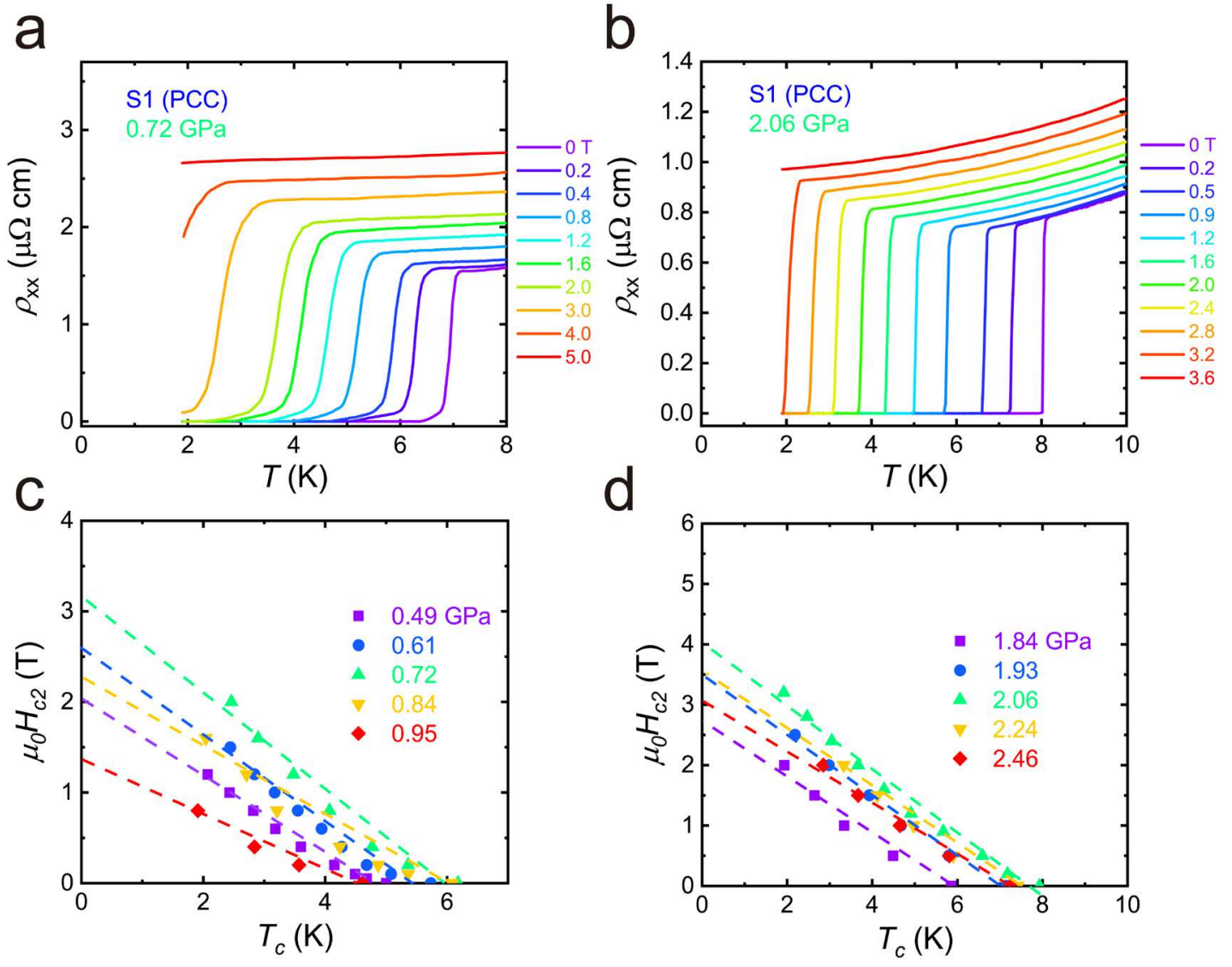


Figure 3.

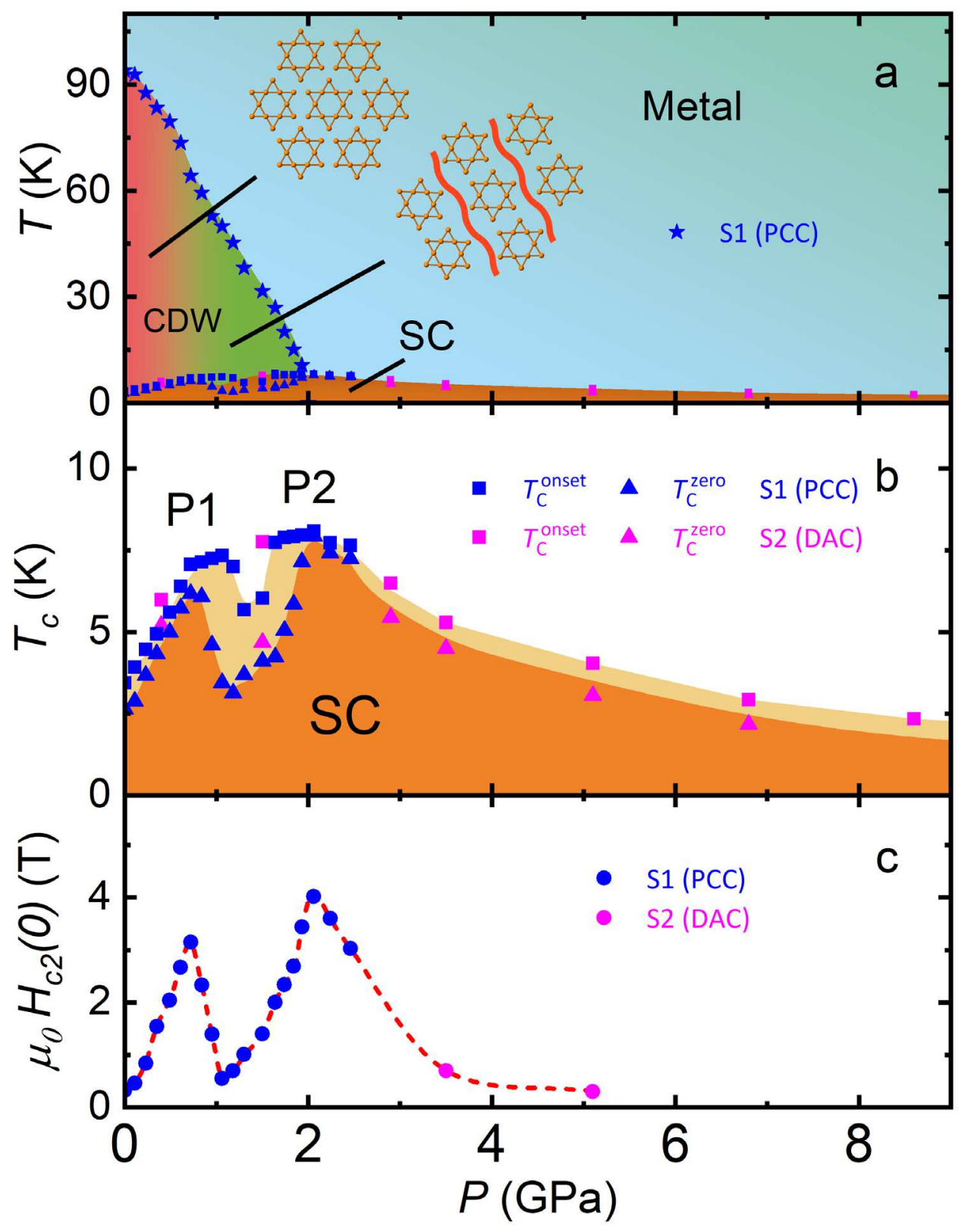


Figure 4.

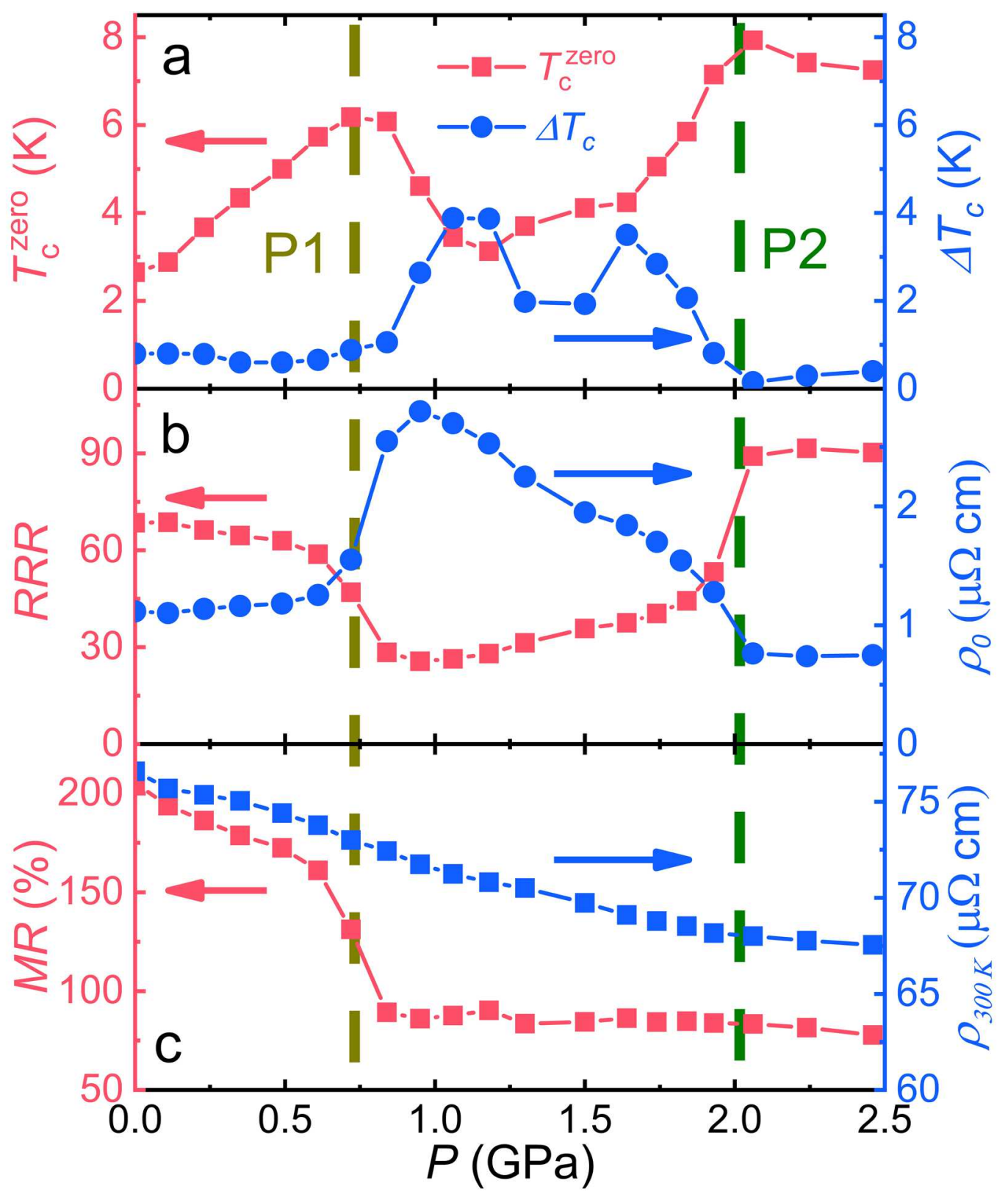




\section{Figures}
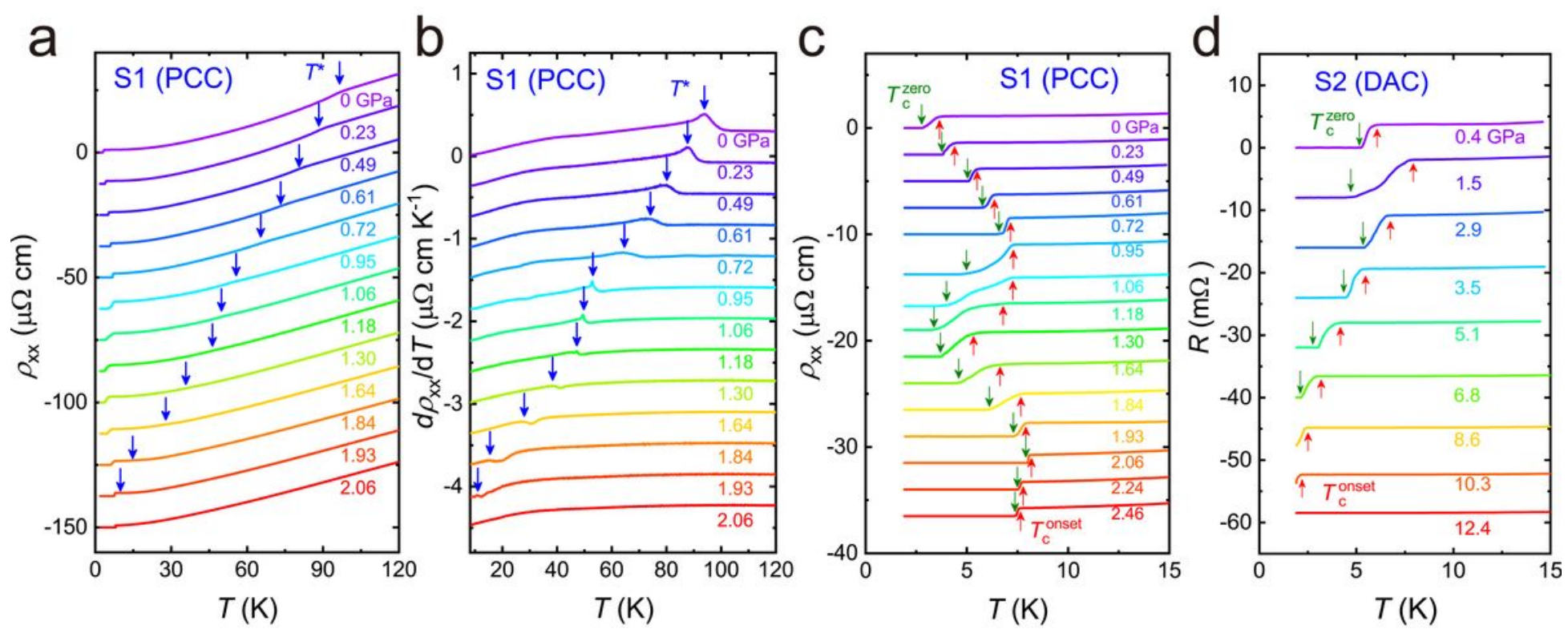

Figure 1

Temperature dependence of resistivity in CsV3Sb5 single crystals under high pressure. (a): Temperature dependences of resistivity under various pressures for sample 1 measured with PCC. (b): The derivative $\mathrm{dpxx} / \mathrm{dT}$ curves under various pressures. The blue arrows indicate the CDW transition temperature $T^{*}$. (c) and (d): The evolution of superconducting transition temperatures under pressure for sample 1 with PCC and sample 2 with DAC. The red and green arrows represent the $T_{-} c^{\wedge}$ onset and $T_{-} c^{\wedge} z e r o$, respectively. All the curves were shifted vertically for clarity. 

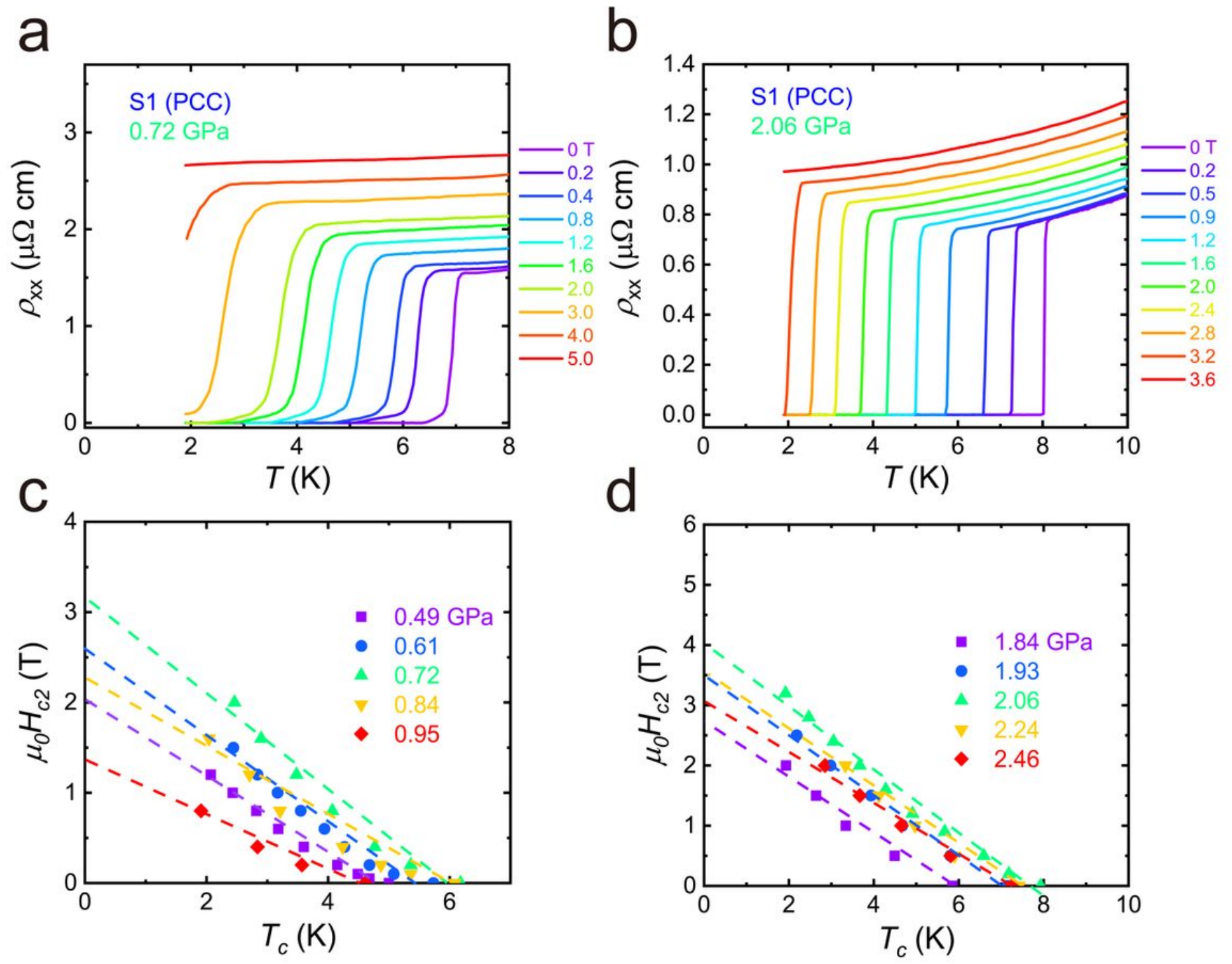

Figure 2

Upper critical field for CsV3Sb5 single crystal under various pressures. Upper critical field (Hc2) measurements for sample1 around P1 (a) and P2 (b) with magnetic field applied along c axis. (c) and (d): The upper critical field $\mu \mathrm{OHc} 2$ vs. Tc under various pressures. 


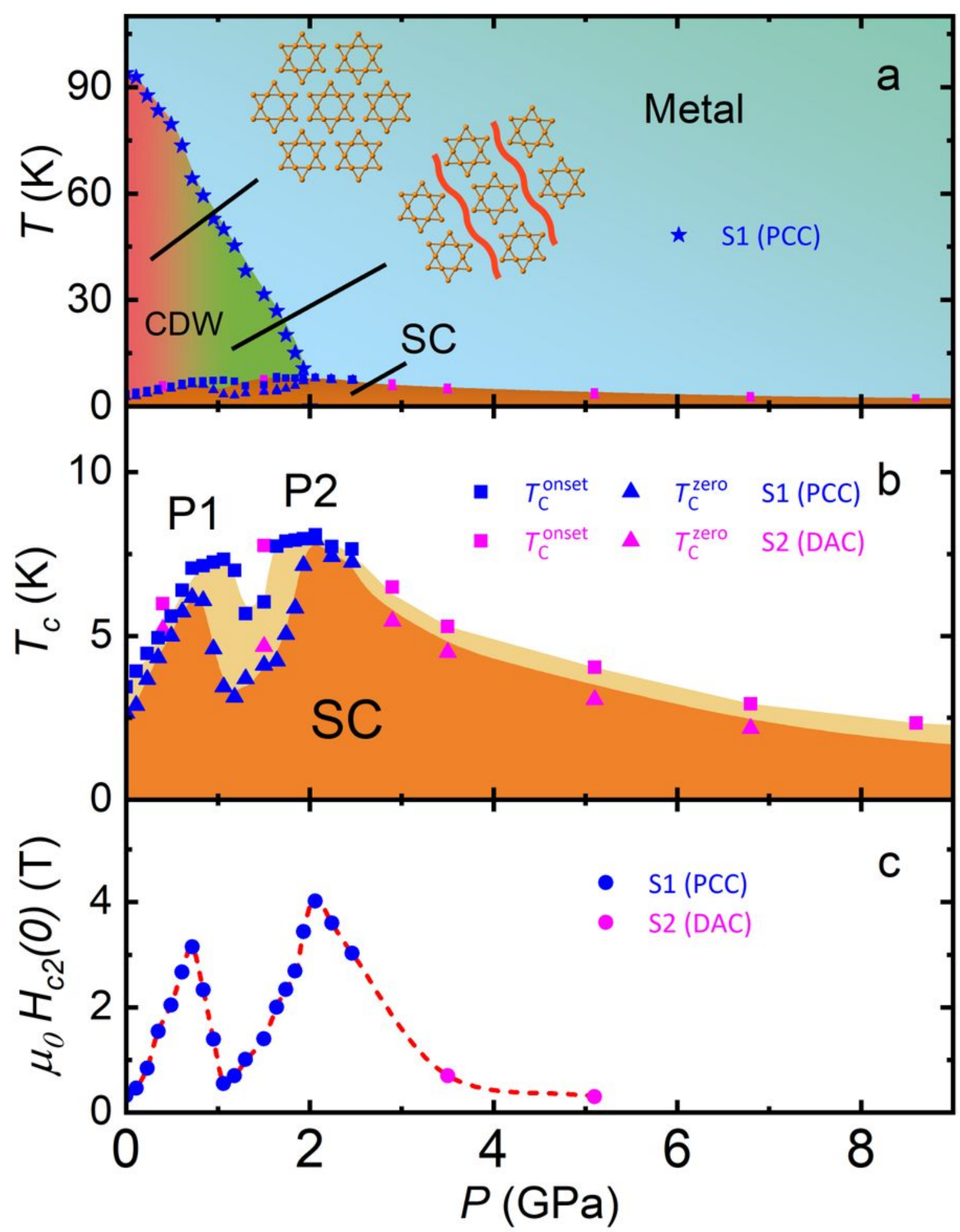

Figure 3

Phase diagram with pressure for CsV3Sb5 single crystal. (a): Phase diagram of CsV3Sb5 with pressure. CDW transition temperature $T^{*}$ gradually suppressed with increasing the pressure. $T$ * shows an anomaly around P1. The inset indicates the probable deformation patterns in CCDW and NCCDW states as discussed in the text. (b): Pressure dependence of superconducting transition temperatures T_c^onset, T_c^zeromeasured on various samples. Tc is strongly suppressed with the pressure between P1 and P2. 
(c): Pressure dependence of upper critical field at zero temperature, two peaks can be observed at P1 and P2.

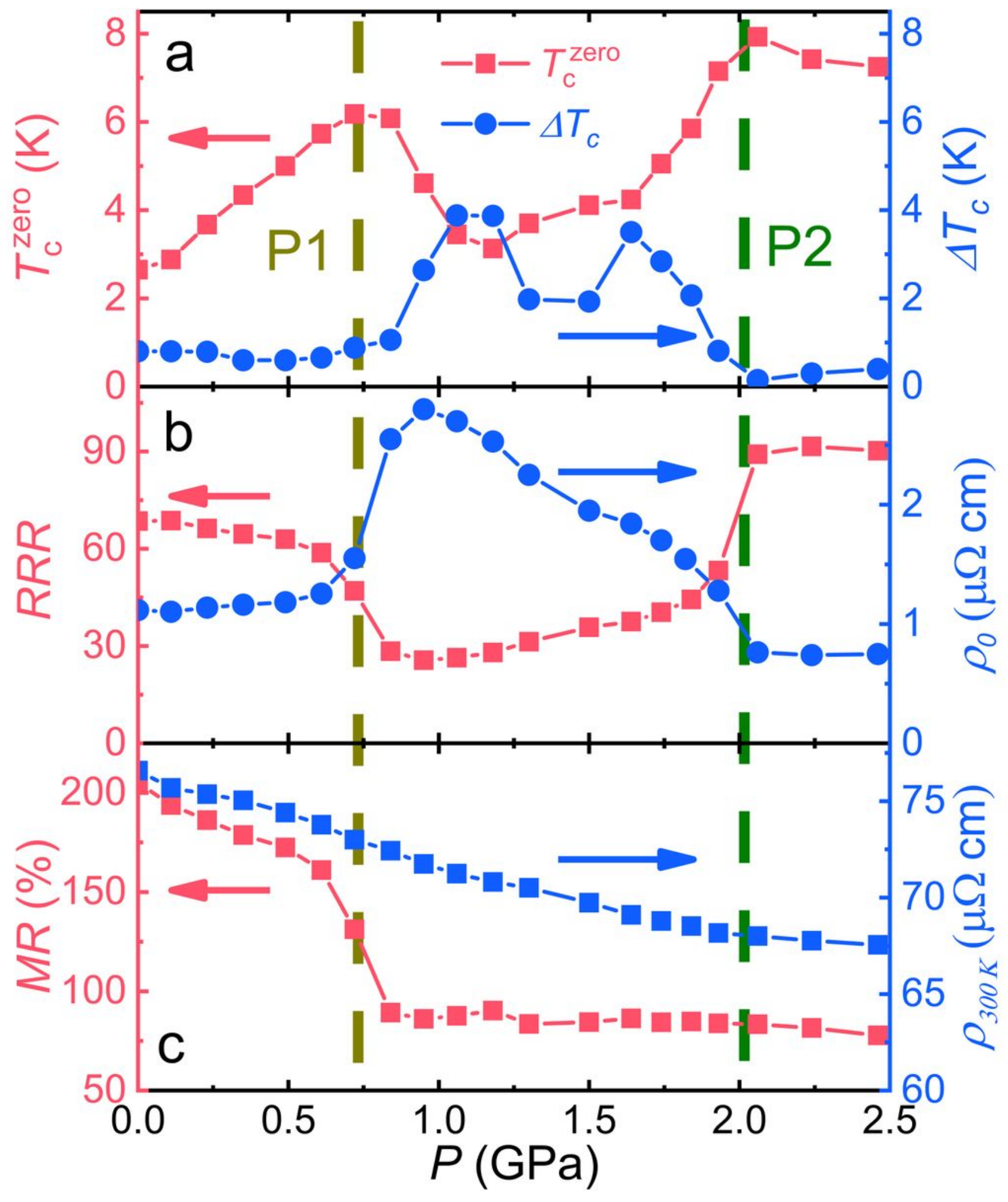

Figure 4

Pressure dependence of residual resistivity, residual-resistivity ratio and magnetoresistance for CsV3Sb5 single crystal. (a): Pressure dependence of T_c^zero and $\triangle T c$ for sample 1 with PCC. T_c ${ }^{\wedge} z e r o$ exhibits two peaks at P1 and P2. (b): Pressure dependence of residual resistivity and residual resistivity ratio 
(RRR) for sample 1 with PCC. (c): Pressure dependence of magnetoresistance (9 T, $10 \mathrm{~K}$ ) and roomtemperature resistivity.

\section{Supplementary Files}

This is a list of supplementary files associated with this preprint. Click to download.

- CsVSbSI.docx 